\title{
TWO YEAR UV-OPTICAL MONITORING OF THE SEYFERT 1
}

\author{
GALAXY MARKARIAN 335
}

\author{
WEI-HSIN SUN ${ }^{1,2}$, CHRIS R. SHRADER ${ }^{2,3}$, TRACEY J. TURNER ${ }^{4}$, \\ MATTHEW A. MALKAN ${ }^{5}$, BRADLEY M. PETERSON ${ }^{6}$, PAUL M.N. HINTZEN ${ }^{7}$, \\ YOJI KONDO ${ }^{8}$, SUNG-NAN LIN ${ }^{1}$, TING-CHANG LIN ${ }^{1}$ and \\ REMINGTON P.S. STONE ${ }^{9}$ \\ ${ }^{1}$ Inst. of Astron. and Dept. of Phys., National Central Univ., Chung-Li, Taiwan S2054, ROC \\ ${ }_{2}$ Guest Observer with the International Ultraviolet Explorer Satellite \\ ${ }^{3}$ GRO Science Support Center, NASA/GSFC, Code 668.1, Greenbelt, MD 20771, USA \\ ${ }^{4}$ Laboratory for High Energy Astrophysics, NASA/GSFC, Code 665, Greenbelt, MD 20771, USA \\ 5 Dept. of Astro., UCLA, Los Angeles, CA 90024, USA \\ ${ }^{6}$ Dept. of Astron., Ohio State Univ., Columbus, OH 49210, USA \\ 7 Dept. of Phys., Univ. of Nevada, Las Vegas, NV 89154, USA \\ ${ }^{8}$ Lab. for Astron. and Solar Phys., NASA/GSFC, Code 684, Greenbelt, MD 20771, USA \\ ${ }^{9}$ Lick Obs., Univ. of California, Mt. Hamilton, CA 95140, USA
}

We report on the results of a UV-Optical spectral monitoring of the bright Seyfert 1 galactic nuclei Mkn 335. This campaign began in June, 1989, and ended in June, 1991. Ultraviolet spectra of fourteen epochs at nearly uniform sampling of 30-day intervals, except when the object was inaccessible from the IUE satellite, have been obtained, of which twelve were coordinated with quasi-simultaneous ground-based optical observations made at Lick Observatory.

Continuum variation at $20-30 \%$ have been observed in both the UV and the optical. Cross-Correlation analysis of line and continuum variability showed that $\mathrm{H}_{\beta}$ lags behind the UV continuum by approximately 30 days while $\mathrm{H}_{\alpha}$ lags behind the UV continuum by 44 days. We fit the multi-epoch UV-Optical continua with (i) a series of power-laws with different slopes and strength, and (ii) the improved accretion disk models. The single power-law in general fits the continua well and we used these fits to derive the emission line properties. In accretion disk fitting, we used a fixed black hole mass, obtained from the averaged spectrum, and varying accretion rate. These single rate accretion disk models provide acceptable fits to almost all the spectra. A series of accretion rates have been inferred which indicated a factor of two variation in rates between the high and low states. The integrated power-law energy correlates well with that estimated from accretion rate. We also found marginal continuum color variation as the luminosity changes. However, the spectral index does not become harder monotonically when the object brightens, in contradiction to what has been suggested previously. We argue that this behavior may be an observational test for hypotheses of the nature of the AGN variability. This work is supported by the National Science Council in Taiwan, ROC, through the grant NSC 82-0208-M-008-011.

Subject headings: galaxies: individual (Mkn 335) - galaxies: Seyfert - quasars: emission lines

T. J.-L. Courvoisier and A. Blecha: Multi-Wavelength Continuum Emission of AGN, 408.

(C) 1994 IAU. Printed in the Netherlands. 